\title{
Acute Macular Neuroretinopathy following Oral Intake of Adrenergic Flu Treatments
}

\author{
Dinu Stanescu-Segall ${ }^{\mathrm{a}}$ Chong Yap ${ }^{\mathrm{b}}$ Ben J.L. Burton ${ }^{\mathrm{c}}$ \\ ${ }^{a}$ Department of Ophthalmology, UZ Leuven, Leuven, Belgium; ${ }^{\text {b} E p s o m ~ a n d ~ S t ~ H e l i e r ~ N H S ~}$ \\ Trust, Epsom, UK; ' James Paget University Hospital NHS Foundation Trust, Great \\ Yarmouth, UK
}

\section{Keywords}

Acute macular neuroretinopathy · Oral intake of adrenergic flu treatment - Optical coherence tomography $\cdot$ Flu treatment

\begin{abstract}
Purpose: The purpose of this study was to report a case series of patients with acute macular neureretinopathy in which patients concomitantly used ephedrine or pseudo-ephedrine. Methods: This is a retrospective case review. Results: Two patients with retinal findings consistent with acute macular neuroretinopathy are presented. Conclusion: Acute macular neuroretinopathy should be considered in any young patient with unexplained loss of vision and exposed to direct or indirect adrenergic stimulation.

(C) 2018 The Author(s)

Published by S. Karger AG, Basel
\end{abstract}

\section{Introduction}

Acute macular neuroretinopathy (AMN) is a rare retinal condition that was first described by Bos and Deutman [1]. The original description consisted of 4 patients who presented with paracentral scotoma, slightly decreased visual acuity, and reddish-brown wedge-shaped intraretinal lesions surrounding the fovea. We describe 2 patients with AMN diagnosed by infrared reflectance and optical coherence tomography (OCT), both of whom were exposed to adrenergic drugs used for treatment of flu-like symptoms before the onset of AMN. 


\section{Case Report}

\section{Case 1}

A 21-year-old female patient presented with scotoma in both eyes. She took Lemsip ${ }^{\circledR} 1$ day before the onset for flu-like symptoms. Her past medical history was normal. Her vision was 6/9 in both eyes. Central visual fields disclosed scotomas corresponding to the patches she described (Fig. 1, 2). Fundoscopy disclosed typical wedge-shaped brownish discoloration in the left macular region (Fig. 3). One week later, her vision improved to 6/5 in both eyes.

\section{Case 2}

A 42-year-old woman presented with a 3-day history of loss of vision in the left eye and bilateral scotoma. Four days before the onset, she had started taking oral pseudoephedrine 60 mg q.d.s. for flu-like symptoms. Her visual acuity was $6 / 5$ and $6 / 24$ in the right and left eye, respectively. Anterior segment examination was normal in both eyes. Fundoscopy revealed reddish brown discoloration around the macular region in both eyes. Infrared pictures disclosed a wedge-shaped lesion on the inter-papillomacular region in the right eye (Fig. 4) and a wedge-shaped lesion encompassing the macula in the left eye (Fig. 5). After 1 month, her vision improved to $6 / 6$ in both eyes with persistent scotomas.

\section{Discussion}

Several conditions have been linked to AMN, including use of oral contraceptive [2], caffeine [3], trauma [4], cocaine [5], preceding flu-like illness [6], and sympathomimetic use, such as adrenaline and epinephrine [7]. To our knowledge, these are the first 2 cases of AMN following solely oral intake of either pseudoephedrine and phenylephrine hydrochloride.

We reviewed the literature and found only 1 patient who took NyQuil D, a solution consisting of acetaminophen, dextromethorphan, doxylamine succinate, and pseudoephedrine, before developing AMN [6]. Our first patient took $60 \mathrm{mg}$ of pseudoephedrine q.d.s. for 4 days before developing her first symptoms. We speculate that she took in total around $1 \mathrm{~g}$ of pseudoephedrine. Our second patient was on Lemsip ${ }^{\circledR}$, a drug sold over the counter, composed of $650 \mathrm{mg}$ of paracetamol and $10 \mathrm{mg}$ of phenylephrine hydrochloride.

These 2 cases reinforce the adrenergic hypothesis involved in the pathogenesis of AMN. Epinephrine can decrease the blood supply to the retina, which has been reported to cause either transient ischemia or permanent changes in several cases. It has also been hypothesized that epinephrine has a direct interactive effect on rhodopsin in the photoreceptor cells of the macula resulting in an immunoreaction at the photoreceptor level [6].

As many as $50 \%$ of patients presenting with AMN have been described with a preceding flu-like syndrome. This percentage is pretty high, and we believe that a more thorough questionnaire might have found intake of drugs, such as pseudoephedrine and phenylephrine, considered as cold remedies that might have been overlooked

We also found it very striking that most of the cases of AMN involved women. In view of the adrenergic hypothesis, it is tempting to make a link with the cerebral stroke occurring more frequently in young women taking oral contraceptives. It is possible that estrogens play a role in the vascular changes found in AMN patients.

A Japanese team found that systemic steroids increased the choroidal blood flow at the site of AMN together with improvement of visual acuity, although the number of patients was small [8]. 
Why so few patients presenting with flu-like syndromes or exposure to adrenergic drugs develop AMN remains a question mark. We speculate that there may be many patients with mild AMN not involving the fovea that remain undiagnosed and that there is likely to be an additional factor which makes some patients much more susceptible to chorioretinal vascular injuries than others.

Red-free photographs and confocal scanning laser ophthalmoscopy infrared reflectance $(810 \mathrm{~nm})$ should be used to be able to detect the typical wedge-shaped lesion surrounding the macular area in cases where AMN is suspected [9]. Spectral-domain OCT shows 1 or more abnormal findings, including ellipsoid zone disruption, hyperreflectivity of the outer nuclear layer, thinning of the outer nuclear layer, hyperreflectivity of the outer plexiform layer, hyporeflectivity of the photoreceptor/retinal pigment epithelium complex, and generalized outer retinal loss. Type 1 lesions, also known as paracentral acute middle maculopathy, localize above the outer plexiform layer with inner layer involvement and eventually resolve with inner nuclear layer atrophy. Conversely, type 2 lesions localize below the outer plexiform layer, with outer macular involvement, resulting finally in outer nuclear atrophy [10].

Disruption of the ellipsoid layer is a permanent change allowing a retrospective diagnosis. These cases highlight the importance of directly asking about over-the-counter medication in this group, as some people may not consider these types of symptomatic treatments as medication and be unaware of the pharmacological nature of the active ingredients.

AMN remains a rare disease. We suggest that AMN should be considered in any young patient with unexplained loss of vision, particularly following exposure to adrenergic stimulation. Nevertheless, it is impossible to dissociate whether adrenergic drug treatment of the viral prodrome has a direct link with AMN. Patients with AMN should be asked about medication use and specifically about over-the-counter flu remedies. Steroids can be considered in the acute phase, but no treatment so far has been proven to improve the vision of these patients.

\section{References}

Bos PJ, Deutman AF: Acute macular neuroretinopathy. Am J Ophthalmol 1975;80:573-584.

Rifkin L, Schaal S: Teenage acute macular neuroretinopathy. Eur J Ophthalmol 2012;22:674-676.

Kerrison JB, Pollock SC, Biousse V, Newman NJ: Coffee and doughnut maculopathy: a cause of acute central ring scotomas. Br J Ophthalmol 2000;84:158-164.

4 Nentwich MM, Leys A, Cramer A, Ulbig MW: Traumatic retinopathy presenting as acute macular neuroretinopathy. Br J Ophthalmol 2013;97:1268-1272.

5 Introini U, Casalino G, Querques G, Bagini M, Bandello F: Acute macular neuroretinopathy following intranasal use of cocaine. Acta Ophthalmol 2015;93:e239-e240.

-6 Aziz HA, Kheir WJ, Young RC, Isom RF, Dubovy SR: Acute macular neuroretinopathy: a case report and review of the literature, 2002-2012. Ophthalmic Surg Lasers Imaging Retina 2015;46:114-124.

-7 Shah SP, Goren JF, Lazzara MD, Strominger MB, Hedges TR, Duker JS, et al: Acute macular neuroretinopathy associated with the use of norepinephrine reuptake inhibitors: a case series and OCT findings. Retin Cases Brief Rep 2013;7:146-149.

8 Hirooka K, Saito W, Noda K, Ishida S: A patient with acute macular neuroretinopathy and central retinal vein occlusion. Clin Ophthalmol 2013;7:1447-1450.

-9 Tingsgaard LK, Sander B, Larsen M: Enhanced visualisation of acute macular neuroretinopathy by spectral imaging. Acta Ophthalmol Scand 1999;77:592-593.

10 Sarraf D, Rahimy E, Fawzi A, et al: Paracentral acute middle maculopathy: a new variant of acute macular neuroretinopathy associated with capillar ischemia. JAMA Ophthalmol 2013;131:1275-1287. 


\section{Case Reports in Ophthalmology}
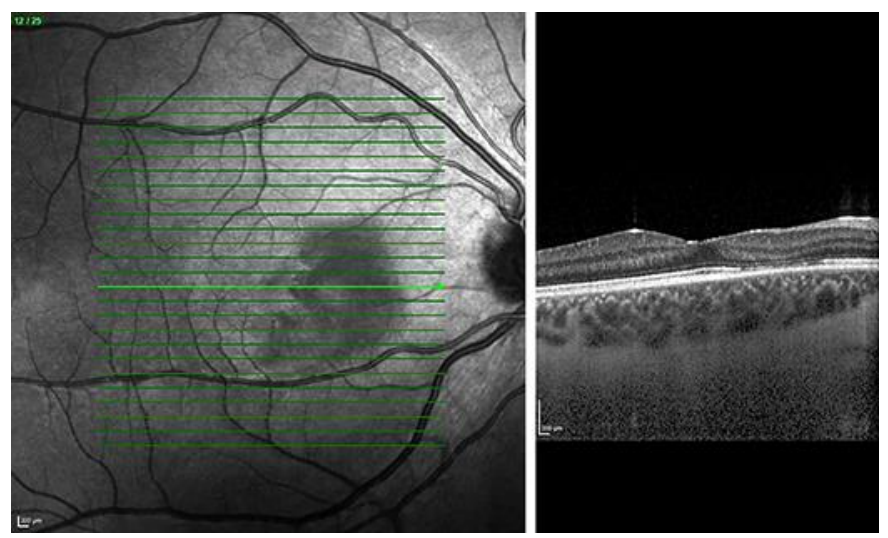

Fig. 1. Right eye (patient 1). Infrared picture showing a large lobulated hyporeflective lesion of 4 DA (disc area) nasal to the fovea. The SD-OCT is disclosing a thinning of the retina with predominantly loss of tissue in the outer nuclear layer and the ellipsoid zone.
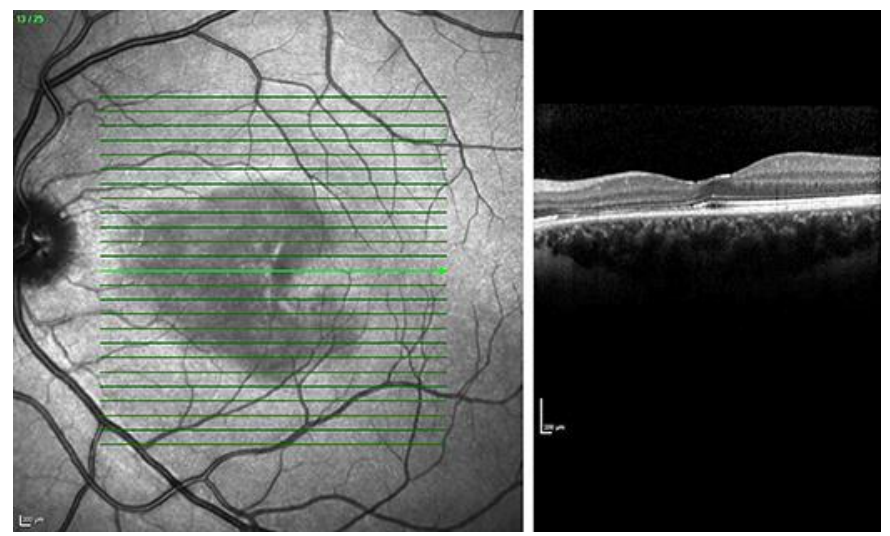

Fig. 2. Left eye (patient 1). Infrared picture showing a large lobulated hyporeflective lesion of 9 DA (disc area) encompassing the fovea and extended nasally. The SD-OCT is disclosing a thinning of the retina with predominantly loss of tissue in the outer nuclear layer and the ellipsoid zone. 


\section{Case Reports in Ophthalmology} www.karger.com/cop

Stanescu-Segall et al:: Acute Macular Neuroretinopathy following Oral Intake of Adrenergic Flu Treatments
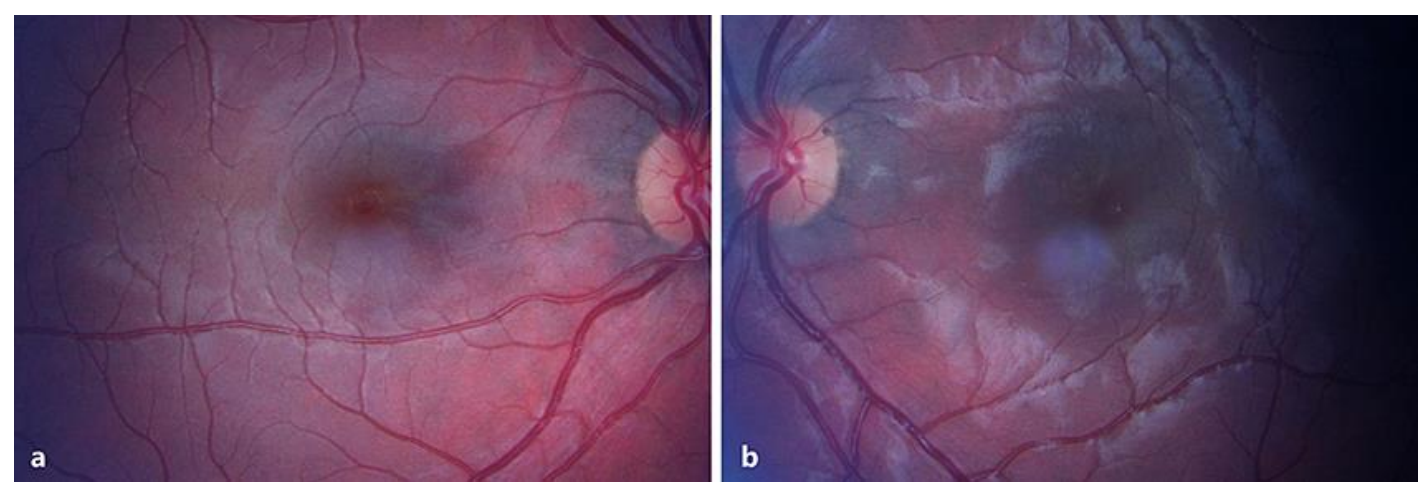

Fig. 3. Patient 1. a Right eye. b Left eye. Color photograph of the right and left macula showing brownish discoloration of the affected retina.
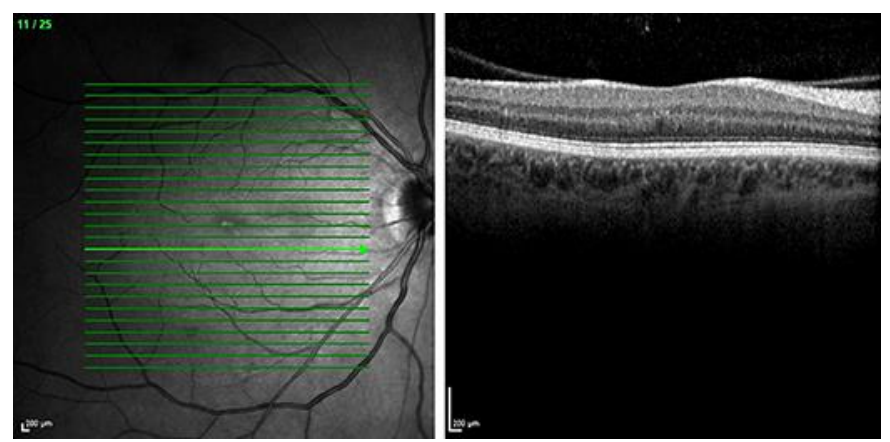

Fig. 4. Right eye (patient 2). Infrared picture showing minor changes of reflectance nasal to the fovea. SDOCT is disclosing white dots in the outer plexiform layer.
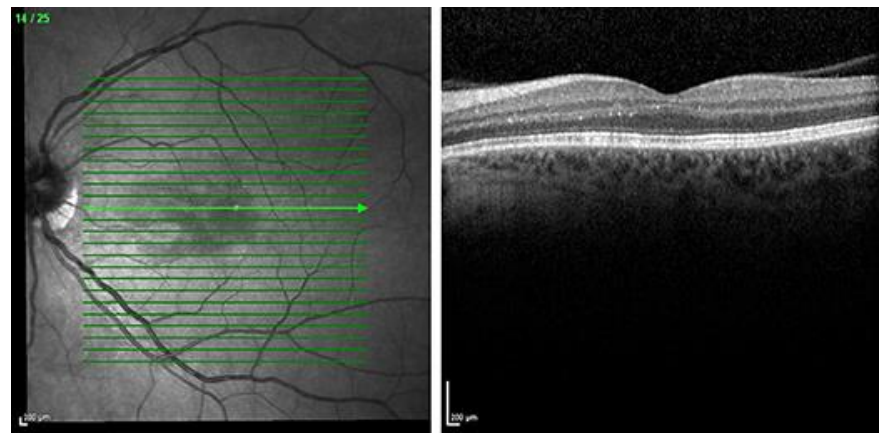

Fig. 5. Left eye (patient 2). Infrared picture showing a lobulated hyporeflective lesion of 4 DA (disc area) involving the fovea and extending nasally. SD-OCT is disclosing white dots in the outer nuclear and outer plexiform layer corresponding to areas of the retina with disrupted ellipsoid zone. 